ARTICLE

\title{
Room-temperature liquid diffused separation induced crystallization for high-quality perovskite single crystals
}

Fang Yao (i) ${ }^{1}$, Jiali Peng ${ }^{1}$, Ruiming $\mathrm{Li}^{1}$, Wenjing $\mathrm{Li}^{1}$, Pengbin Gui ${ }^{1}$, Borui $\mathrm{Li}^{1}$, Chang $\mathrm{Liu}^{1}$, Chen Tao (i) ${ }^{1 凶}$, Qianqian Lin ${ }^{1 凶} \&$ Guojia Fang (1) ${ }^{1 凶}$

Large single crystals serve as an ideal platform for investigating intrinsic material properties and optoelectronic applications. Here we develop a method, namely, room-temperature liquid diffused separation induced crystallization that uses silicone oil to separate the solvent from the perovskite precursors, to grow high-quality perovskite single crystals. The growth kinetics of perovskite single crystals using this method is elucidated, and their structural and optoelectronic properties are carefully characterized. The resultant perovskite single crystals, taking $\mathrm{CH}_{3} \mathrm{NH}_{3} \mathrm{PbBr}_{3}$ as an example, exhibit approximately $1 \mu$ s lifetime, a low trap density of $4.4 \times 10^{9} \mathrm{~cm}^{-3}$, and high yield of $92 \%$, which are appealing for visible light or X-ray detection. We hope our findings will be of great significance for the continued advancement of highquality perovskite single crystals, through a better understanding of growth mechanisms and their deployment in various optoelectronics. The diffused separation induced crystallization strategy presents a major step forward for advancing the field on perovskite single crystals.

\footnotetext{
${ }^{1}$ Key Lab of Artificial Micro- and Nano-Structures of Ministry of Education of China, School of Physics and Technology, Wuhan University, 430072

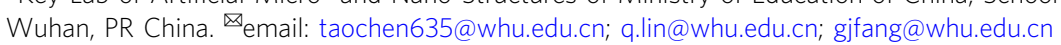


rganic-inorganic trihalide perovskites $\mathrm{APbX}_{3} \quad(\mathrm{~A}=$ $\mathrm{CH}_{3} \mathrm{NH}_{3}{ }^{+}, \mathrm{H}_{2} \mathrm{NCHNH}_{2}{ }^{+}, \mathrm{Cs}^{+} \mathrm{X}=\mathrm{Cl}^{-}, \mathrm{Br}^{-}, \mathrm{I}^{-}$) have made tremendous progress in optoelectronics, e.g., solar cells ${ }^{1-5}$, light-emitting diodes ${ }^{6-8}$, lasers, and photodetectors $(\mathrm{PDs})^{9-14}$. This is attributed to their excellent optoelectronic properties, e.g., high absorption coefficients over tunable and wide wavelength range, ambipolar charge transport with long and balanced electron-hole diffusion lengths, high carrier mobility, and large dielectric constant ${ }^{15,16}$. Perovskite polycrystalline thin films can be easily deposited with low-temperature solution processes with lost cost regardless of either flat or wrinkled substrates ${ }^{13,17,18}$. Compared with its polycrystalline films, perovskite single crystals (SCs) present none grain boundaries, lower recombination centers, and trap density ${ }^{19-21}$. Moreover, they are more environmentally stable 22 . As a semiconductor crystalline material, SCs are frequently employed as an ideal platform for investigating their optoelectronic properties ${ }^{23-26}$.

Up to now, large hybrid perovskite SCs have been prepared with various solution-based methods, including inverse temperature crystallization (ITC) ${ }^{27,28}$, temperature lowering $\operatorname{method}^{29}$, top-seeded solution-growth method ${ }^{20}$, antisolvent vapor-assisted crystallization $(\mathrm{AVC})^{19}$, Bridgman growth method ${ }^{30}$, cavitationtriggered asymmetrical crystallization (CTAC), and lowtemperature-gradient crystallization (LTGC) ${ }^{25,31}$. These growth routes for perovskite SCs have made great progress. Optoelectronic applications based on SCs, e.g., solar cells 23,32 , detectors $11,12,25,33,34$, have exhibited superior performances. Bakr and coworkers reported perovskite SC solar cells with power conversion efficiencies reaching up to $21 \%^{32}$, Liu et al. fabricated a PD based on perovskite SCs with detectivity as high as $10^{13}$ Jones $^{25}$. Huang and coworkers reported high radiation and imaging detection devices with sensitivity up to $2.1 \times 10^{4} \mu \mathrm{C} \mathrm{Gy}{ }_{\text {air }}{ }^{-1} \mathrm{~cm}^{-2}$ under $8 \mathrm{KeV} \mathrm{X}$-ray radiation with perovskite $\mathrm{SCs}^{35,36}$. Among these approaches, a rapid facile crystallization route based on the "inverse solubility" effect has been widely used ${ }^{27,29,37}$. It produced perovskite SCs with $100 \mathrm{~mm}$ in several hours, one order of magnitude faster than the other methods ${ }^{19,20,38}$. However, the way to obtain supersaturated perovskite solutions in those methods is upon either temperature or antisolvent. The convective currents arising from thermal gradients in growth solution inevitably disturb the ordered growth, leading to undesired twining defects and cracks in the bulk of perovskite SCs ${ }^{19,39,40}$. Moreover, the solubility difference upon controlling temperature is limited ${ }^{19}$. In addition, perovskite SCs grown at low temperatures present much lower trap density than those at high temperatures ${ }^{37}$. Therefore, high-quality perovskite SCs grown at a constant, low temperature are highly desired.

In this work, we develop a novel room-temperature (RT) liquid-diffused separation induced crystallization (LDSC) method to grow high-quality 3-dimentional (3D) $\mathrm{CH}_{3} \mathrm{NH}_{3} \mathrm{PbX}_{3}$ $\left(\mathrm{MAPbX}_{3}, \mathrm{X}=\mathrm{Cl}, \mathrm{Br}, \mathrm{I}\right)$ and 2-dimentional (2D) $\left(\mathrm{C}_{4} \mathrm{H}_{9} \mathrm{NH}_{3}\right)_{2}$ $\mathrm{PbBr}_{4}\left(\mathrm{BA}_{2} \mathrm{PbBr}_{4}\right),\left(\mathrm{C}_{3} \mathrm{H}_{7} \mathrm{NH}_{3}\right)_{2} \mathrm{PbBr}_{4}\left(\mathrm{PA}_{2} \mathrm{PbBr}_{4}\right)$, and $\left(\mathrm{C}_{6} \mathrm{H}_{5}\right.$ $\left.\mathrm{CH}_{2} \mathrm{NH}_{3}\right)_{2} \mathrm{PbBr}_{4}\left(\mathrm{PMA}_{2} \mathrm{PbBr}_{4}\right)$ perovskite SCs. The crystallization process initializes from an unsaturated perovskite solution by changing the solution concentration, that is, the solvent escapes out of the solution through spontaneously diffusing into silicone oil. When it reaches saturated, we keep the concentration of the perovskite precursor as a constant and only change the volume in the following process. We select silicone oil as the separation medium as its density is slightly higher than that of solvent but lower than that of perovskite precursor. The solvent diffuses into the silicone oil and escapes out, leading to the formation of oversaturated perovskite precursor solutions. In contrast to controlling the heating or cooling rate, maintaining the precursor solution at RT is apparently much easier. With this knowledge, we establish this facile crystallization route for growing SCs across a wide range of perovskite semiconductors and uncover how to enable to grow high-quality perovskite SCs at RT. We further elucidate the growth kinetics mechanism via the LDSC method in detail. The thickness of the bulk 3D SCs can be tuned from microns to millimeters. Compared with the high-temperature $\mathrm{MAPbBr}_{3}\left(\mathrm{HT}-\mathrm{MAPbBr}_{3}\right)$ ones, the LDSC$\mathrm{MAPbBr}_{3}$ SC detectors show a lower dark current, faster response speed, higher photocurrent, and X-ray sensitivity. The versatility of our approach provides a generic strategy to grow high-quality perovskite SCs with the selection of suitable solvents and medium materials, which further pushes towards nextgeneration perovskite optoelectronic applications.

\section{Results}

Growth kinetics of perovskite SCs. It is noted that the growth temperature and precursor concentration play key roles in determining both the quality and the yield of the resultant SCs ${ }^{25}$. Since solubility is measured in terms of the maximum amount of solute dissolved in a solvent at equilibrium, a solution system can reach oversaturated by varying temperature and concentration. The driving force of crystallization from a metastable perovskite precursor solution is oversaturation, which is determined by its temperature and concentration ${ }^{41}$. Typically, the saturation concentration is essentially dependent on temperature ${ }^{37}$. The saturated concentration of perovskite precursor solutions decreases upon increasing temperature, that is, inverse solubility ${ }^{27,29}$. This has been frequently taken for growing perovskite SCs, which is well known as the ITC method. In our case, however, the volume of the precursor solutions shrinks while the concentration of the perovskite precursors keeps as a constant. The absence of temperature fluctuation leads to better SC quality as demonstrated below.

Figure 1a schematically illustrates the growth diagram of perovskite SCs at RT. The solvent of the precursor solution spontaneously diffuses into silicone oil. In the meanwhile, the precursor solution evolves to be more concentrated. Then perovskite SCs appear in the oversaturated solution. The thickness of crystals can be tailored by tuning the height of the precursor solution from microns to millimeters (Supplementary Fig. 1). To obtain high-quality SCs, the crystal growth should be maximized and extra nucleation should be suppressed. Besides the $\mathrm{MAPbBr}_{3}$ one (shown in Fig. 1d), we obtained $\mathrm{MAPbCl}_{3}, \mathrm{MAPbI}_{3} 3 \mathrm{D}$ perovskite SCs, and $\mathrm{BA}_{2} \mathrm{PbBr}_{4}$, $\mathrm{PA}_{2} \mathrm{PbBr}_{4}$ and $\mathrm{PMA}_{2} \mathrm{PbBr}_{4} 2 \mathrm{D}$ layered perovskite $\mathrm{SCs}$ using the LDSC method, as shown in Supplementary Fig. 2. The X-ray $2 \theta$ scan on the facet of the crystal presents diffraction peaks, suggesting a well-orientated $\mathrm{MAPbCl}_{3}, \mathrm{MAPbI}_{3}, \mathrm{BA}_{2} \mathrm{PbBr}_{4}, \mathrm{PA}_{2} \mathrm{PbBr}_{4}$, and $\mathrm{PMA}_{2} \mathrm{PbBr}_{4}$ crystalline, as shown in Supplementary Fig. 3, which are in line with literatures $28,42-44$

In a metastable solution system, the crystal growth rate is determined by the deposition rate and diffusion rate. The deposition rate is mainly controlled by the concentration and temperature of the solution, while the diffusion rate is by the concentration gradient of the solution. The crystal seed growth is determined by the deposition rate. To prepare high-quality SCs, the deposition rate should be less than the diffusion rate. When crystal is grown in oversaturation solution, the model can be expressed as ${ }^{25,45}$

$$
-V \frac{\mathrm{d} \Delta C}{\mathrm{~d} t}=V \frac{\mathrm{d} M}{\mathrm{~d} t}
$$

where $V$ is the solution volume, $C$ the constant concentration of the saturated solution, and $M$ the molar weight of crystal, respectively.

In our case, the temperature and concentration of precursor solutions are kept as a constant. Thus, varying the volume of the solution would tune the concentration gradient. Given that the 
a

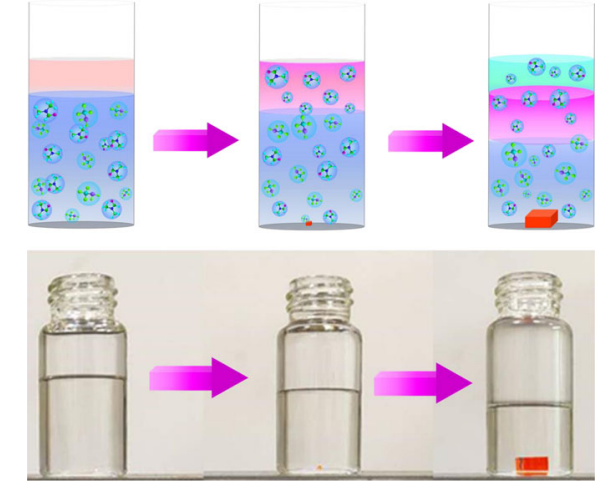

C

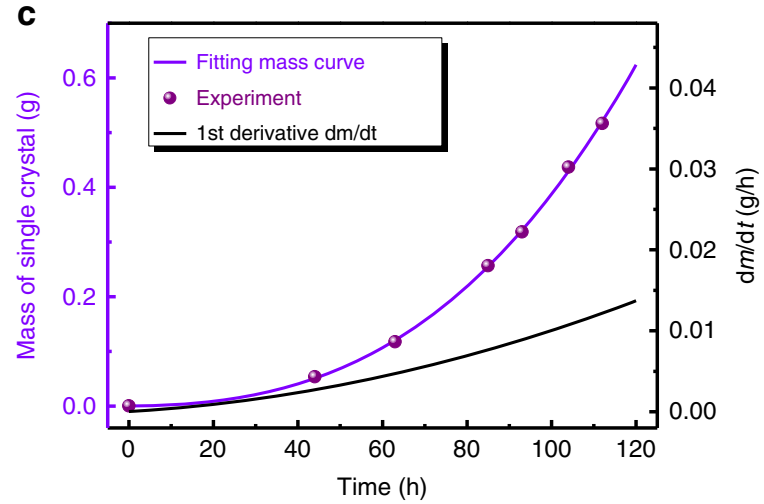

b

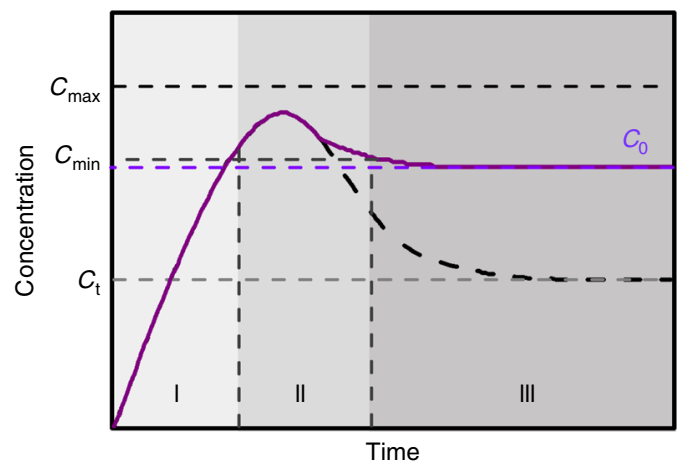

d

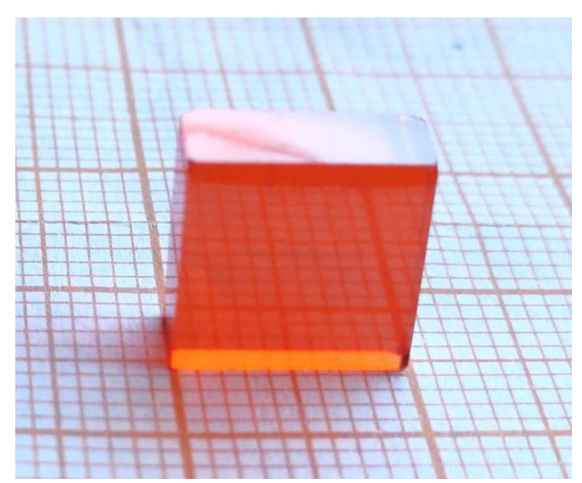

Fig. 1 Perovskite single-crystal growth. a Schematic illustration of growth diagram of perovskite SCs. b Schematic representation of the concentration of solution before and after nucleation as a function of time. $C_{t}$ is the solubility, $C_{0}$ is the concentration of perovskite solution $\left(C_{0}>C_{t}\right), C_{\text {min }}$ is the minimum concentration for nucleation, i.e., the minimum oversaturation level for nucleation, and $C_{\max }$ is the maximum concentration for nucleation. The regions I, II, and III represent prenucleation, nucleation, and growth stage, respectively ${ }^{48}$. c The mass and mass derivative as a function of the growth time for the $\mathrm{MAPbBr}_{3} \mathrm{SC}$ in DMF with silicone oil as a medium. d Photograph of $\mathrm{MAPbBr}_{3}$ SC grown using the RT LDSC method.

concentration of the saturated solution is constant, the volume of the solution shrinks as $\mathrm{N}, \mathrm{N}$-dimethylformamide (DMF) continues to be extracted. Therefore, the crystal growth rate as a function of the solution volume can be expressed as:

$$
\frac{\mathrm{d} m}{\mathrm{~d} t}=-\frac{\mathrm{d}}{\mathrm{d} t}\left[\left((V(t)-V(0)) \cdot C \cdot M_{\mathrm{MAPbBr}_{3}}\right)-m\right],
$$

where $m$ is the mass of the crystal, $V$ the solution volume, $C$ the constant concentration of the saturated solution, and $M_{\mathrm{MAPBBr}_{3}}$ the molar weight of $\mathrm{MAPbBr}_{3}$, respectively. This LaMer model had been reported to present the formation process of the monodispersed solution ${ }^{46,47}$. In Fig. 1b, the processes of nucleation and growth through the LaMer diagram comprise three stages. At the first stage of prenucleation, DMF diffuses into silicone oil and escapes out, while the monomeric perovskite species initialize to accumulate in solution. Thus, no appreciable nucleation occurs, even when the concentration exceeds the solubility level $\left(C_{t}\right)$ of the perovskite precursor. At the second stage of nucleation, the concentration reaches a critical minimum level $\left(C_{\min }\right)$ for nucleation and subsequently the nucleation commences. At the third stage of maximum oversaturation, the nucleation dramatically accelerates as the concentration reaches the maximum level $\left(C_{\max }\right)$. The accumulation and consumption of the perovskite precursor are balanced at the crystal nucleation and growth stages. The crystal growth terminates when the concentration of monomers is close to $C_{\mathrm{t}}$ (Fig. 1b, black dash line $)^{48}$. Note that DMF still keeps diffusing at this stage, in spite of neither accumulation nor consumption of the perovskite precursors. Thus, the precursor concentration is constant once the nucleation occurs (Fig. $1 \mathrm{~b}$ purple solid line). $C_{0}$ is always larger than $C_{\mathrm{t}}$ when the perovskite solution is oversaturated. The variation of $C_{0}$ during crystal growth is negligible. Equation (2) can thus be simplified as

$$
\frac{\mathrm{d}}{\mathrm{d} t}=-\frac{1}{2} C \cdot M_{\mathrm{MAPBBr}} \cdot \frac{\mathrm{d} V(t)}{\mathrm{d} t},
$$

To figure out the relationship of mass of crystal versus growth time, the mass of crystal and growth time were recorded. In Fig. 1c, the curve of crystal growth was obtained by fitting the experimental data. As a result, the mass derivative with respect to the growth time was calculated. It is worth noted that the rate of crystal growth significantly increases upon the growth time.

We next move to model the seed crystal growth. The crystal growth model is determined by two steps: mass transfer and surface integration. In this model, the solute is transferred from the solution to the crystal interface by the mass transfer process, followed by the solute being integrated into the crystal lattice in the surface integration step. Each step can be described as ${ }^{45,49,50}$

$$
\begin{aligned}
& -\frac{\mathrm{d} m}{\mathrm{~d} t}=r_{\mathrm{c}} \cdot A_{\mathrm{s}} \cdot\left(C-C_{\mathrm{i}}\right), \\
& -\frac{\mathrm{d} m}{\mathrm{~d} t}=r_{\mathrm{i}} \cdot A_{\mathrm{s}} \cdot\left(C_{\mathrm{i}}-C_{\mathrm{t}}\right)^{k},
\end{aligned}
$$

where $r_{\mathrm{c}}$ is the mass transfer coefficient, $A_{\mathrm{s}}$ the surface area of SC, $C$ and $C_{\mathrm{i}}$ the solute concentration in solution and at the crystal interface, respectively, $r_{\mathrm{i}}$ the surface integration coefficient, $C_{\mathrm{t}}$ the solubility of solute, and $k$ the surface integration order. Combining Eqs. (4) and (5), the two-step growth model for a SC can be 
rearranged as $\left(\Delta C=C-C_{t}\right)$ :

$$
\Delta C=\left(-\frac{\mathrm{d} m}{\mathrm{~d} t} \frac{1}{r_{\mathrm{i}} S}\right)^{\frac{1}{k}}-\frac{\mathrm{d} m}{\mathrm{~d} t} \frac{1}{r_{\mathrm{c}} S},
$$

when $\Delta C=0$ (the concentration is unchanged), the growth rate can be expressed as

$$
\frac{\mathrm{d} m}{\mathrm{~d} t}=\left(-\frac{\left(r_{\mathrm{c}}\right)^{k}}{r_{\mathrm{i}}}\right)^{\frac{1}{k-1}} \cdot S=K \cdot S,
$$

where $K$ is a constant as the temperature is always at RT. Therefore, the growth rate of SCs is proportional to their surface area. In Fig. 1c, it is obvious that $\mathrm{dm} / \mathrm{dt}$ sharply increases upon the growth time. In this case, we know that the surface area of crystal is as a quadratic function of time. Combining with the concentration function, the theoretical and measured crystal yield with respect to the growth concentration are calculated and illustrated in Supplementary Fig. 4. We find that the yield at high concentrations is larger than that at low concentrations. The yield reaches up to $92 \%$ at $0.5 \mathrm{~mol} \mathrm{~L}^{-1}$, which is the highest reported value so far. Figure $1 \mathrm{~d}$ displays a $\mathrm{MAPbBr}_{3} \mathrm{SC}$ obtained using the RT LDSC method. The thickness of the crystals can be tailored from microns to millimeter $(38 \mu \mathrm{m}$ to $4 \mathrm{~mm})$ as shown in Supplementary Fig. 1a-g.

Characterization of $\mathrm{MAPbBr}_{3}$ SCs. The crystallographic structure of $\mathrm{MAPbBr}_{3}$ SC grown using the RT LDSC method was investigated with $\mathrm{X}$-ray diffraction (XRD). For comparison, $\mathrm{MAPbBr}_{3}$ SCs using the HT method ${ }^{27}$ are also grown and investigated. In Fig. $2 \mathrm{a}$, the XRD $2 \theta$ scan on its maximal facet presents only (100) and (200) diffraction peaks, suggesting a well-orientated
$\mathrm{MAPbBr}_{3}$ crystalline. The phi scan curve of the (201) diffraction peak shows that the rotation of the (201) diffraction peak gives four peaks with a $90^{\circ}$ interval (Fig. 2b). It is further ascertained that the crystal is quadruple symmetric. Then, the (100) and (200) peaks were further analyzed carefully using high-resolution X-ray rocking curve with fixed $2 \theta$ at $14.96^{\circ}$ of (100) and $30.15^{\circ}$ of (200), respectively (Fig. $2 \mathrm{c}$ ). The full width at half maximum (FWHM) of these two peaks are as narrow as $0.0163^{\circ}$ and $0.0096^{\circ}$ for the (100) and (200) peaks, respectively. These values are much smaller than the HT method prepared crystals, i.e., $0.0183^{\circ}$ and $0.0173^{\circ}$ for the (100) and (200) peaks, respectively. Furthermore, we also compared our LDSC method with the reported LTGC method ${ }^{25}$. Interestingly, we found the same trend, that is, low temperature can effectively reduce the FWHM of rocking curves compared with high-temperature methods, indicating enhanced crystallinity. In addition, the FWHM of rocking curve at (200) is $0.0096^{\circ}$, which is slightly narrower than the record value $\left(0.013^{\circ}\right)$ reported for $\mathrm{MAPbBr}_{3}$ SCs by Liu et al., which may attribute to the further decreased growth temperature of LDSC at RT, compared with the LTGC method at $60^{\circ} \mathrm{C}^{25,51}$. In addition, when the $2 \theta$ was fixed, the sample was azimuthally rotated from $0^{\circ}$ to $360^{\circ}$ at a series of tilted angles from $0^{\circ}$ to $90^{\circ}$. The appearance of discrete spots in the pole figure instead of rings indicates that the $\mathrm{MAPbBr}_{3} \mathrm{SC}$ is in order both out-of-plane and in-plane. Figure $2 \mathrm{~d}$ shows a pole figure of $\mathrm{MAPbBr}_{3} \mathrm{SC}$ with spots (in red rings) azimuthally separated with $90^{\circ}$.

Next, we move to investigate the optical properties of the $\mathrm{MAPbBr}_{3}$ SCs. Supplementary Fig. 5a presents the absorption spectra of $\mathrm{LDSC}^{-\mathrm{MAPbBr}_{3}}$ and $\mathrm{HT}-\mathrm{MAPbBr}_{3}$ SCs. Both $\mathrm{MAPbBr}_{3} \mathrm{SCs}$ exhibit a sharp absorption onset at $595 \mathrm{~nm}$. The optical band gap $(2.15 \mathrm{eV})$ was estimated from the photoluminescence (PL) and absorption spectra, which is in line with

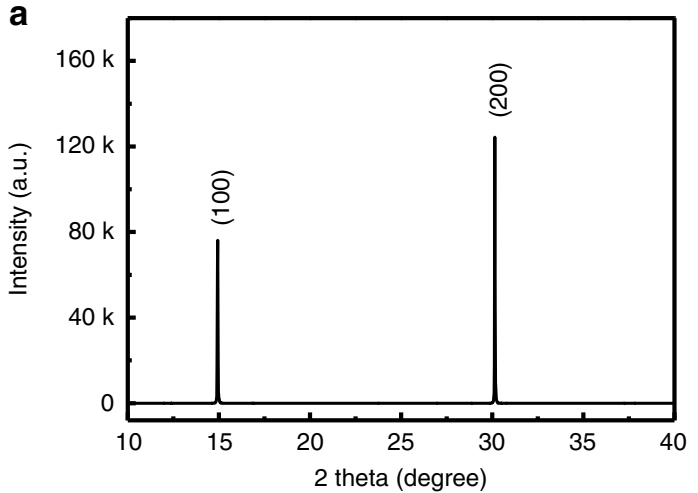

C

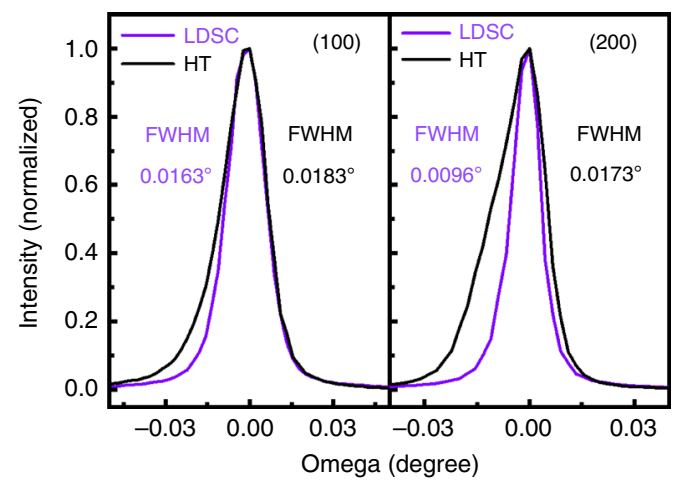

b

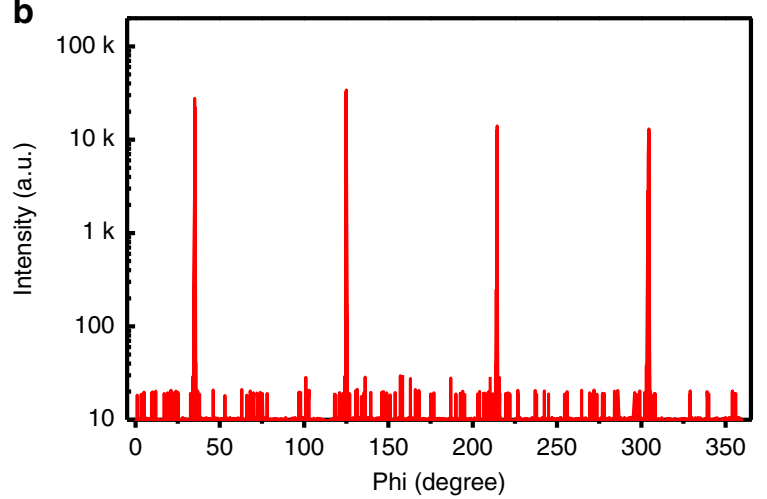

d

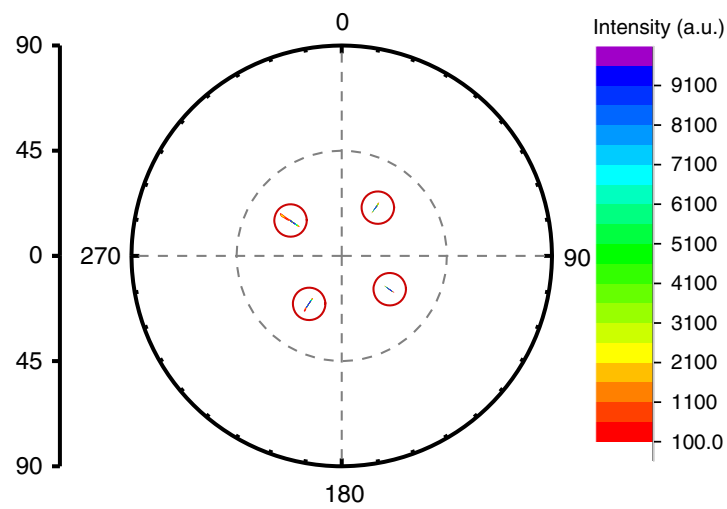

Fig. 2 X-ray diffraction of $\mathbf{M A P b B r}_{\mathbf{3}}$ single crystals. a XRD $2 \theta$ scans of the $\mathrm{MAPbBr}_{3} \mathrm{SC}$ grown using the LDSC method. b Phi scan curve of the (201) diffraction peak of the $\mathrm{MAPbBr}_{3} \mathrm{SC}$. c High-resolution XRD rocking curve of the (100) and (200) diffraction peaks of the $\mathrm{MAPbBr}_{3} \mathrm{SCs}$ grown using the LDSC and HT methods. $\mathbf{d}$ The pole figure of the (201) diffraction peak of the $\mathrm{MAPbBr}_{3}$ SC. 
a
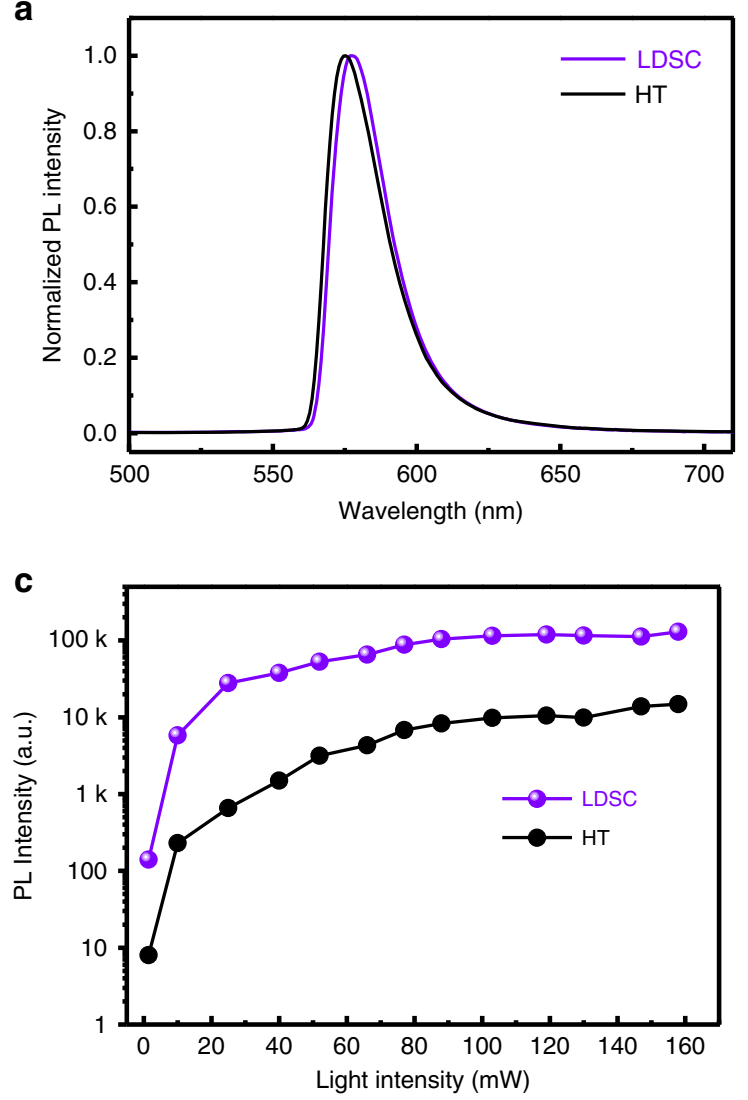

b
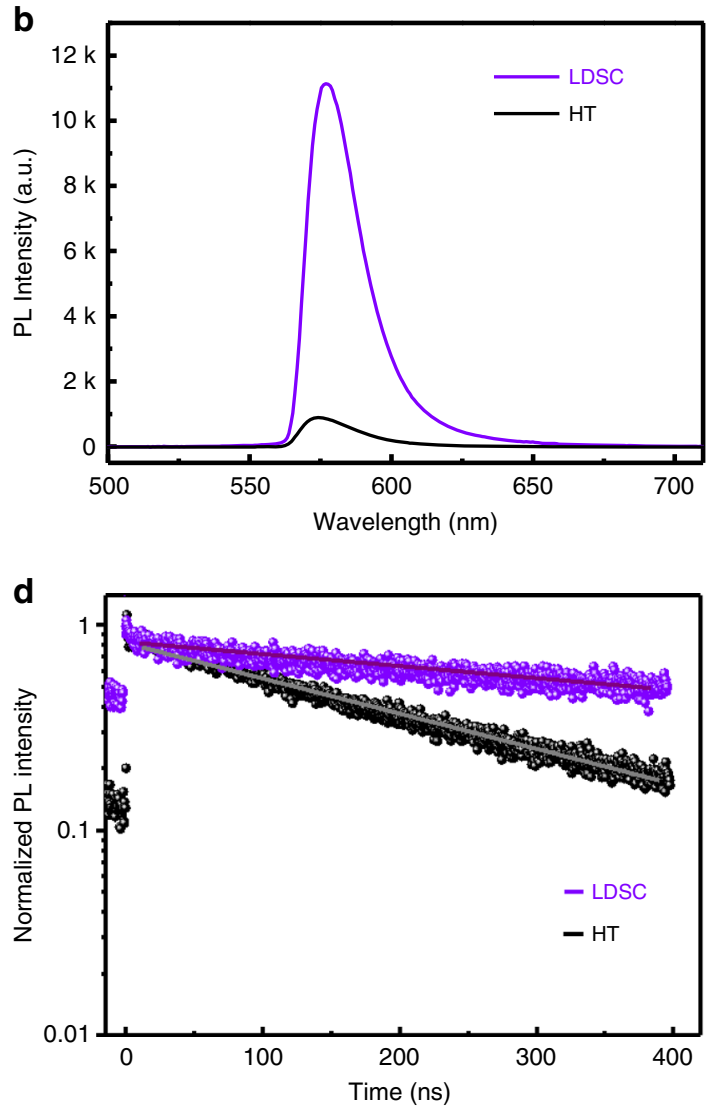

Fig. 3 Photoluminescence of $\mathbf{M A P b B r}_{3}$ single crystals. a Normalized photoluminescence (PL) spectra of $L D S C-M A P b B r_{3}$ and $H T-M A P b B r_{3} S C$. b PL spectra of LDSC-MAPbBr 3 and $\mathrm{HT}-\mathrm{MAPbBr}_{3} \mathrm{SC}$ at the same excitation intensity. c PL intensity of LDSC-MAPbBr 3 and $\mathrm{HT}-\mathrm{MAPbBr}_{3} \mathrm{SCs}$ as a function of excitation intensity. d Time-resolved photoluminescence of the LDSC-MAPbBr 3 (violet) and the HT-MAPbBr 3 (dark) SCs.

literatures ${ }^{38}$. The absorption coefficient $\alpha$ was calculated by the absorption spectrum and reflectance spectrum (Supplementary Fig. 6). In the long wavelength region, as shown in Supplementary Fig. 5b, the $a$ of LDSC-MAPbBr 3 SC is smaller than that of HT$\mathrm{MAPbBr}_{3} \mathrm{SC}$, indicating weaker subband gap absorption and lower trap density.

The PL peaks of LDSC-MAPbBr ${ }_{3}$ and HT-MAPbBr 3 SCs locate at $577 \mathrm{~nm}$, with a narrow FWHM of $22 \mathrm{~nm}$ (Fig. 3a). However, the PL intensity of both crystals exhibited substantial difference at the same excitation intensity. As shown in Fig. 3b, the PL intensity of $\mathrm{LDSC}-\mathrm{MAPbBr}_{3}$ is more than ten times higher than HT$\mathrm{MAPbBr}_{3}$, which means that the quality of LDSC-MAPbBr 3 SC is higher than that of HT-MAPbBr 3 SC. Furthermore, we investigated the PL intensity as a function of excitation intensity for both SCs. As shown in Fig. 3c, the PL intensity increases rapidly upon the increase of light intensity. The PL intensity of LDSC-MAPbBr SC is promptly increased and becomes saturated, while the HT$\mathrm{MAPbBr}_{3}$ keeps increasing. We further verified it from the PL intensity derivative as a function of excitation intensity, which can be seen in Supplementary Fig. 7. The results indicate that the nonradiative recombination of the LDSC-MAPbBr 3 SC is much lower than that of $\mathrm{HT}-\mathrm{MAPbBr}_{3} \mathrm{SC}$. The recombination dynamics of photoexcited carriers in both LDSC-MAPbBr 3 and HT$\mathrm{MAPbBr}_{3}$ SCs were investigated with time-resolved photoluminescence (TRPL) spectroscopy (Fig. 3d). The mono-exponential lifetimes can be calculated from the mono-exponential fitting to the PL decay. The LDSC-MAPbBr ${ }_{3}$ SC exhibits significantly longer carrier lifetime (997 ns) in contrast to that of the HT-MAPbBr 3 SC $(235 \mathrm{~ns})$, indicating much lower monomolecular recombination rates and fewer defects. These results demonstrate that superior quality is achieved for $\mathrm{MAPbBr}_{3}$ SCs using our LDSC strategy.

We investigated the key metrics of perovskite SCs that directly affect their deployment in optoelectronic applications: carrier lifetime $\tau$, and trap density. The trap density $\left(n_{\text {traps }}\right)$ of the $\mathrm{MAPbBr}_{3}$ SCs were investigated by the space-charge-limited current (SCLC) method. Typically, the electron-only device structure was $(\mathrm{Cu} / \mathrm{C} 60 / \mathrm{SC} / \mathrm{C} 60 / \mathrm{Cu})$, while the hole-only one was $\mathrm{Au} / \mathrm{SC} / \mathrm{Au}$. Figure 4 displays the dark $\mathrm{I}-\mathrm{E}$ characteristics of HT$\mathrm{MAPbBr}_{3}$ SCs (Fig. 4a, b) and LDSC-MAPbBr 3 SCs (Fig. 4c, d), respectively. Two regions were divided in the experimental data. At low bias voltages, the $I-E$ response was ohmic (i.e., linear), as confirmed by the fit to linear dependence (green line). At intermediate bias voltages, a trap-filled region started at $V_{\mathrm{TFL}}$ where the current exhibited a rapid nonlinear rise and signaled the transition into the trap-filled limit (TFL) - a regime in which all the available trap states were filled by the injected carriers. The trap density $\left(n_{\text {traps }}\right)$ was calculated using the following relation ${ }^{43}$ :

$$
n_{\text {traps }}=\frac{2 V_{\mathrm{TFL}} \varepsilon \varepsilon_{0}}{e L^{2}},
$$

where $V_{\mathrm{TFL}}$ is the TFL voltage, $\varepsilon$ the relative dielectric constant of perovskite $(25.5)^{52}, \varepsilon_{0}$ the vacuum permittivity, $e$ the electronic charge, and $L$ the thickness of the crystal. The determined electron trap density is $2.7( \pm 0.2) \times 10^{10} \mathrm{~cm}^{-3}$ for HT-MAPbBr 3 SC, while it is only $6.5( \pm 0.4) \times 10^{9} \mathrm{~cm}^{-3}$ for LDSC-MAPbBr SC. The hole trap density is $2.2( \pm 0.1) \times 10^{10} \mathrm{~cm}^{-3}$ for $\mathrm{HT}-\mathrm{MAPbBr}_{3} \mathrm{SC}$, while it is only $4.4( \pm 0.2) \times 10^{9} \mathrm{~cm}^{-3}$ for 

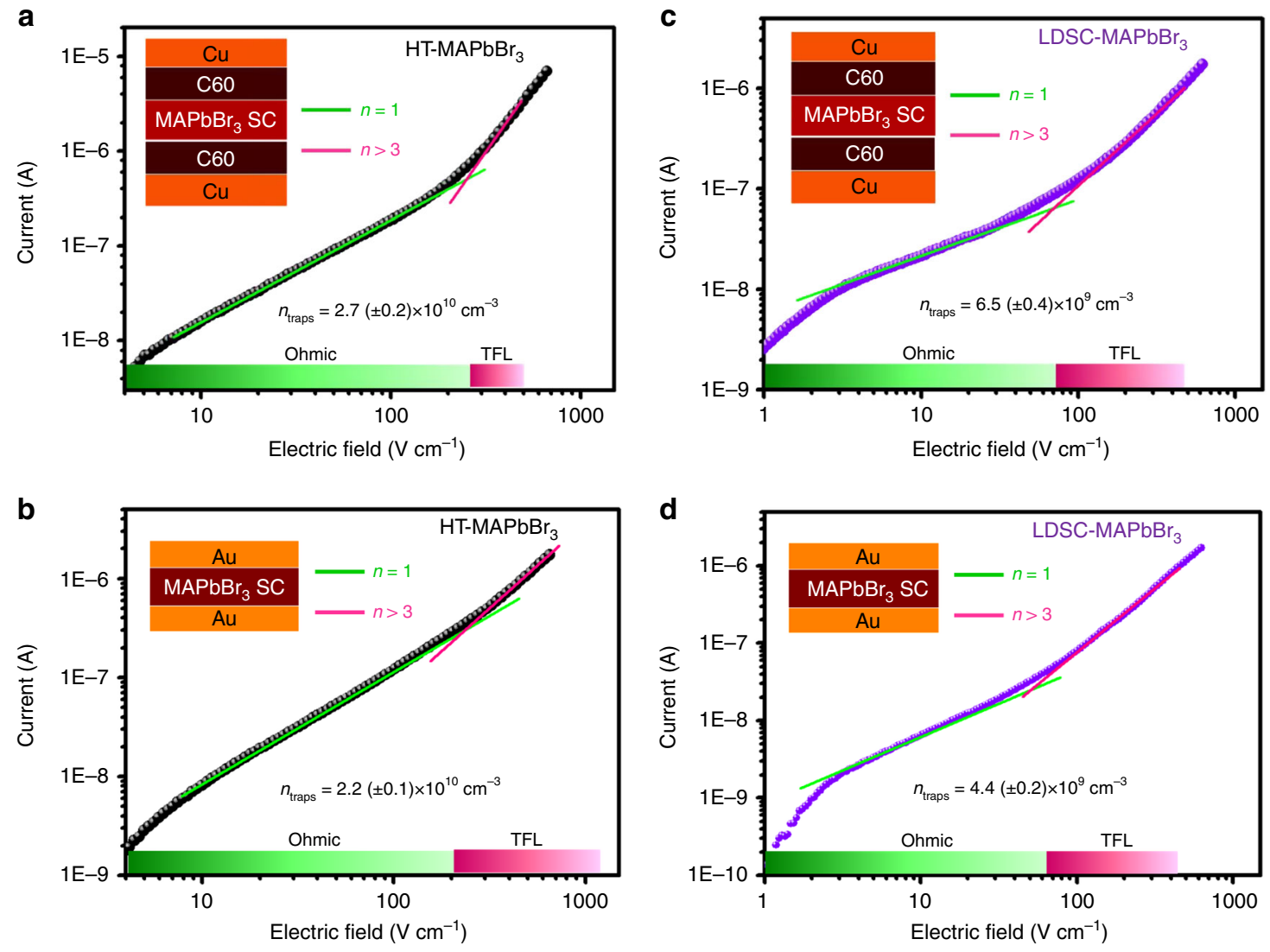

Fig. 4 Characterization of single crystals trap density. Characteristic I-E curves of a electron-only and $\mathbf{b}$ hole-only devices of $\mathrm{HT}-\mathrm{MAPbBr}_{3}$, and c electron-only and $\mathbf{d}$ hole-only devices of $\mathrm{LDSC}-\mathrm{MAPbBr}_{3}$, respectively. A linear ohmic regime (green line) is followed by the trap-filled regime, marked by a steep increase in current (pink line).

Table 1 Performance metrics of $\mathrm{MAPbBr}_{3} \mathrm{SCs}$ with different growth methods.

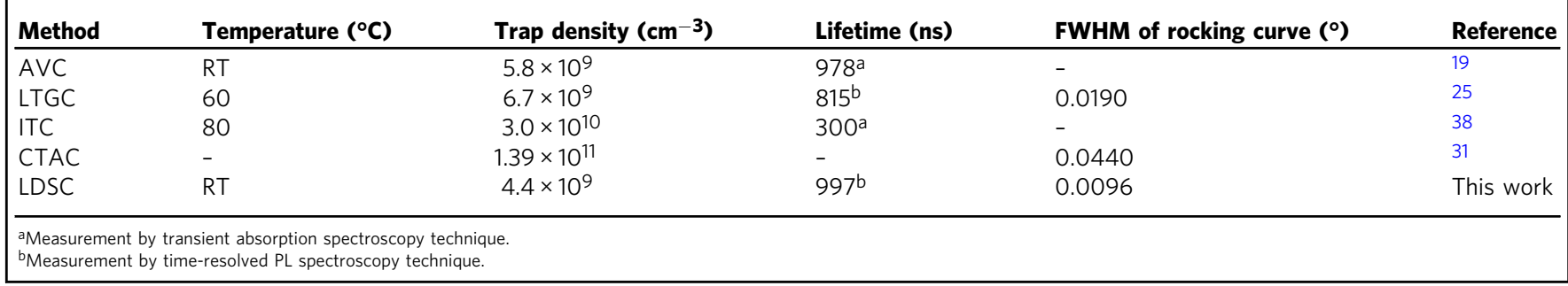

LDSC-MAPbBr 3 SC. Note that the $n_{\text {traps }}$ of the LDSC-MAPbBr 3 $\mathrm{SC}$ is much lower than that of its polycrystalline counterparts $\left(1 \times 10^{17} \mathrm{~cm}^{-3}\right)^{25}$ as well as those of most state-of-theart semiconductors, including $\mathrm{Si}\left(10^{13}-10^{14} \mathrm{~cm}^{-3}\right)^{53}$, CIGS $\left(10^{13} \mathrm{~cm}^{-3}\right)^{54}$, and CdTe $\left(10^{11}-10^{13} \mathrm{~cm}^{-3}\right)^{55}$. The ultralow trap density indicates excellent crystal quality for the LDSC-MAPbBr SC. The essential properties of $\mathrm{MAPbBr}_{3} \mathrm{SC}$ using various methods at different temperatures are summarized in Table 1. One can conclude that our RT LDSC-MAPbBr ${ }_{3}$ SCs are superior in terms of carrier lifetime and trap density, which are essential for high-performance optoelectronic devices. The ultralow trap density is essential to enable a low dark current, which is critical for achieving high-performance perovskite detectors ${ }^{17,56}$.

Characterization of SC-based photodetectors. The electronic properties of perovskite SCs were characterized. The $\mathrm{MAPbBr}_{3} \mathrm{SC}$
PDs with the structure of $\mathrm{InGa} / \mathrm{C} 70 / \mathrm{MAPbBr}_{3} / \mathrm{Au}$ (shown in Fig. 5a) were fabricated. Figure 5a illustrates detector characteristics under the illumination of visible light or X-ray. The $\mathrm{MAPbBr}_{3} \mathrm{SC}$ PDs exhibit an ultralow dark current. As shown in Fig. 5b, the photocurrent of the LDSC-MAPbBr 3 SC PDs under the illumination by white LED is as twice as that of the HT-MAPbBr ${ }_{3}$ SC PDs. The photocurrent at negative bias increases drastically for both SC PDs, indicating that a considerable number of carriers are photogenerated. The sensitivity and lowest detectable dose rate are the most important figures of merit to evaluate the performance of an $\mathrm{X}$-ray detector. As shown in Fig. 5c, d the generated photocurrent density signal has a linear relationship with the X-ray dose rates. The X-ray sensitivity was examined by fitting the photocurrent density as a function of the dose rate. For the 2.02-mm-thick LDSC$\mathrm{MAPbBr}_{3}$ SC-based X-ray detector, a sensitivity of $30.0 \mu \mathrm{C} \mathrm{Gy}{ }^{-1}$ $\mathrm{cm}^{-2}$ under zero bias is obtained, which is almost as five times as that of $1.42-\mathrm{mm}$-thick HT-MAPbBr 3 SC one $\left(6.5 \mu \mathrm{C} \mathrm{Gy}^{-1} \mathrm{~cm}^{-2}\right)$. 
a
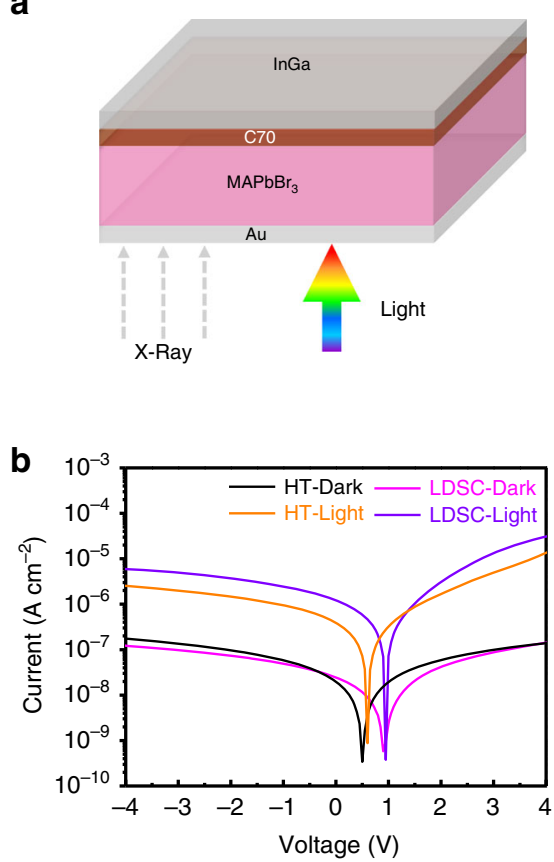

c
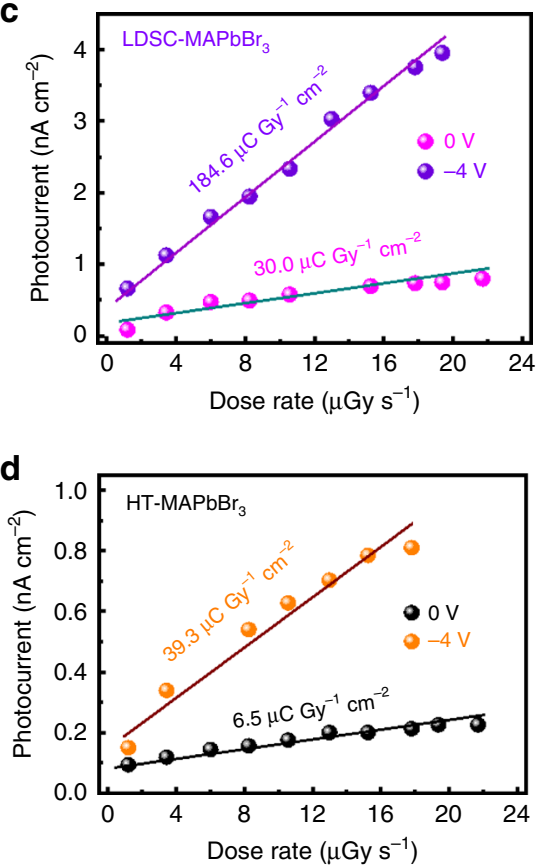

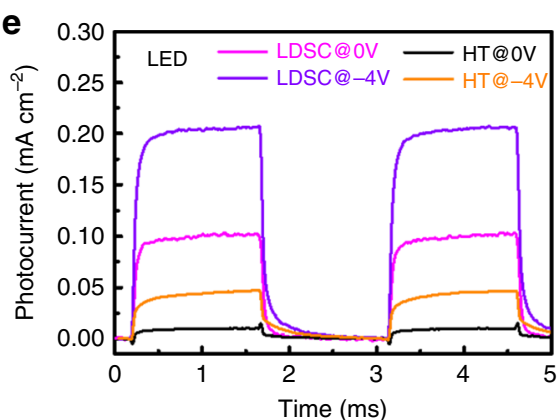

f

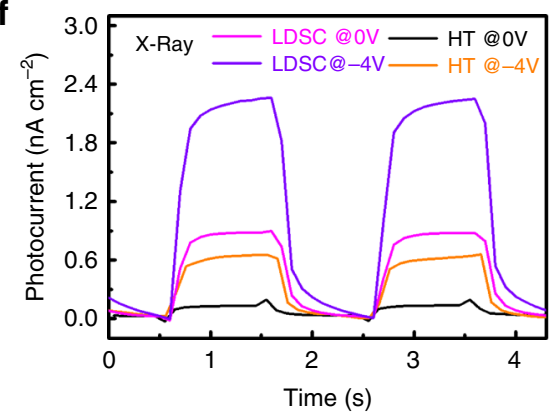

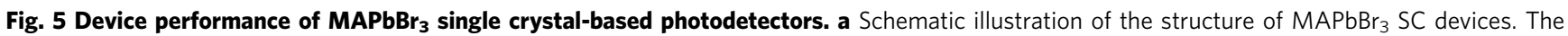
carriers generated under the illumination of visible light or X-ray. $\mathbf{b}$ Dark current and photocurrent of $\mathrm{MAPbBr}_{3} \mathrm{SC}$ devices. $\mathrm{X}$-ray generated photocurrent versus dose rate of $\mathrm{LDSC}-\mathrm{MAPbBr}_{3} \mathrm{SC}$ detector (c) and $\mathrm{HT}-\mathrm{MAPbBr}_{3} \mathrm{SC}$ one (d), respectively. Current response of $\mathrm{MAPbBr}_{3} \mathrm{SC}$ device under the illumination of white LED (e) and X-ray (f) at different biases, respectively.

Furthermore, the LDSC-MAPbBr 3 SC X-ray detector shows a sensitivity of $184.6 \mu \mathrm{C} \mathrm{Gy}^{-1} \mathrm{~cm}^{-2}$ at $-4 \mathrm{~V}$, much higher than that of $\mathrm{HT}-\mathrm{MAPbBr}_{3}$ one $\left(39.3 \mu \mathrm{C} \mathrm{Gy} \mathrm{C}^{-1}\right)$. In particularly, the detectable X-ray dose rate of LDSC-MAPbBr 3 SC X-ray detector is lower than $1.2 \mu \mathrm{Gy} \mathrm{s}^{-1}$, and achieved a high sensitivity of $184.6 \mu \mathrm{C}$ $\mathrm{Gy}^{-1} \mathrm{~cm}^{-2}$, which is comparable with reported $\alpha$-Se X-ray detectors $\left(400 \mu \mathrm{C} \mathrm{Gy}^{-1} \mathrm{~cm}^{-2}\right)^{57,58}$. However, these $\alpha$-Se X-ray detectors are operated with high electrical field of $15 \mathrm{~V} \mathrm{\mu m}^{-1}$, and our devices require relatively low electrical field of $2 \times 10^{-3} \mathrm{~V} \mathrm{~mm}^{-1}$. Figure $5 \mathrm{e}, \mathrm{f}$ shows on/off photocurrent with respect to time at different biases under the illumination of white LED and X-ray irradiation, respectively. From 0 to $-4 \mathrm{~V}$, the photocurrent increase of the LDSC-MAPbBr ${ }_{3} \mathrm{X}$-ray detector is higher than that of the HT$\mathrm{MAPbBr}_{3}$ one. Due to the better charge transport of LDSC$\mathrm{MAPbBr}_{3} \mathrm{SCs}$, the detector at zero bias shows a shorter response time, i.e., the raise and recovery time, of $93 \mu$ s for the device than those of $\mathrm{HT}-\mathrm{MAPbBr}_{3}$ SC one $(206 \mu \mathrm{s})$ under the illumination of white LED, respectively (Supplementary Fig. 8). Similarly, at $-4 \mathrm{~V}$, the LDSC-MAPbBr 3 SC detector shows a relatively shorter response time of $62 \mu$ s compared with $\mathrm{HT}-\mathrm{MAPbBr}_{3}$ one $(205 \mu \mathrm{s})$, respectively. We further evaluate the stability of SC detectors through measuring their photoresponse under modulated light illumination without encapsulation in air after 10,000 working cycles. The LDSC-MAPbBr 3 SC device shows quite stable while the $\mathrm{HT}-\mathrm{MAPbBr}_{3}$ SC device has a little decrease in photoresponse, as shown in Supplementary Fig. 9. The LDSC-MAPbBr 3 SC device shows a lower dark current, faster response speed, higher photocurrent and X-ray sensitivity than those of $\mathrm{HT}-\mathrm{MAPbBr}_{3} \mathrm{SC}$ one. These are ascribed to the longer carrier lifetime, and lower trap density of the LDSC-MAPbBr 3 perovskite, showing superiority optoelectronic applications.

\section{Discussion}

In summary, we report a novel strategy to grow halide perovskite SCs at RT. The perfect combination of solvent selection, silicone oil and growth parameters (e.g., concentration and temperature) allows us to successfully grow various $2 \mathrm{D}$ and $3 \mathrm{D}$ perovskite $\mathrm{SCs}$ using our LDSC method. As the solvent and silicone oil have different density and polarity, they are spontaneously separated from the hybrid precursor solutions. The absence of temperature fluctuation leads to perovskite SCs with ultralow trap density, almost a half of those using the HT method. High-quality $\mathrm{MAPbBr}_{3}$ SCs with excellent crystallinity (FWHM of rocking curve $\left.<0.0096^{\circ}\right)$, low trap density $\left(4.4 \times 10^{9} \mathrm{~cm}^{-3}\right)$, and the highest yield (up to 92\%) was obtained. As a result, the LDSC$\mathrm{MAPbBr}_{3} \mathrm{SC}$ detectors show a lower dark current, faster response speed, higher photocurrent, and X-ray sensitivity. It is expected that the continuous advancement of high-quality perovskite SCs further pushes the development of the new generation of perovskite optoelectronics with superior performance.

\section{Methods}

Chemicals and reagents. n-Butylammonium bromide (BABr), propylammonium Bromide $(\mathrm{PABr})$, and Benzylammonium bromide (PMABr) were purchased from Xi'an Polymer Light Technology Corp. $\mathrm{PbBr}_{2}(98 \%)$ and silicone oil were purchased from Alfa Aesar. DMF, lead iodide $\left(\mathrm{PbI}_{2}\right)$, dimethyl sulfoxide (DMSO), and methylammonium chloride $(\mathrm{MACl})$ were purchased from Sigma Aldrich. Methylammonium bromide (MABr) and methylammonium iodide (MAI) was purchased from Greatcell Solar Materials Pty Ltd. Acetonitrile (ACN) was purchased from Aladdin. All materials were used as received without further purification.

Growth of the MAPbX $\mathbf{M}_{\mathbf{3}}$ perovskite SCs. For the LDSC method, MAX (PABr, $\mathrm{BABr}$, and $\mathrm{PMABr})$, and $\mathrm{PbX}_{2}(\mathrm{X}=\mathrm{Br}, \mathrm{Cl}, \mathrm{I})$ were dissolved at a $1: 1(2: 1)$ molar ratio in DMF (DMF:DMSO =1:1, ACN:GBL (gamma-Butyrolactone) $=6: 4$ ) solvent. To obtain proper dissolution, the solution was maintained at room temperature under stirring overnight, followed by filtration. After adding an appropriate amount of precursor solution and silicone oil, and placed at RT $\left(\mathrm{MAPbI}_{3}\right.$ for $\left.65^{\circ} \mathrm{C}\right)$. It should be noted that the crystalline takes several days at a constant temperature. A large- and high-quality $\mathrm{MAPbX}_{3}$ or $\mathrm{BA}_{2} \mathrm{PbBr}_{4}, \mathrm{PA}_{2} \mathrm{PbBr}_{4}$, and $\mathrm{PMA}_{2} \mathrm{PbBr}_{4}$ SCs were obtained, shown in Fig. 1d and Supplementary Fig. 2.

To grow $\mathrm{SC}$ at high temperature, $\mathrm{MABr}$ and $\mathrm{PbBr}_{2}$ were dissolved in DMF solvent with a 1:1 molar ratio. To obtain a proper dissolution, the solution was maintained at room temperature under stirring overnight, followed by filtration. The precursor solution was then transferred into a clean beaker and placed on a hot plate with rising temperature to $90^{\circ} \mathrm{C}$. The $\mathrm{HT}-\mathrm{MAPbBr}_{3} \mathrm{SC}$ was obtained after several hours. 
High-resolution XRD on perovskite SCs. The high-resolution XRD measurements were taken using a Bede $\mathrm{D} 1$ with $\mathrm{Cu} \mathrm{K}_{\alpha 1}$ radiation $(\lambda=1.54056 \AA)$. The film texture was characterized by XRD $2 \theta$-scans, $\varphi$-scans, omega-scans, and pole figure measurements with $\mathrm{Cu}$ Ka radiation. A pole figure consists of performing a scan about the surface normal ( $\varphi$ scan) with fixed $2 \theta$, fixed $\kappa$, and at a range of $\varphi$ scan. One then rotates the sample about the surface normal while measuring the diffracted intensity in steps of $\varphi$ about $\kappa_{210}$. In this way, we can be absolutely sensitive to the in-plane orientation of the crystal structure.

Absorbance and reflectance spectra measurements. The high-resolution absorption spectra were measured at room temperature with a home-built spectrometer using a monochromator with Xenon lamp as light source. The light intensity was calibrated with certified Si detector, and the photocurrent was recorded with a lock-in amplifier. Reflectance spectra were recorded with an FRBasic-UV/NIR-HR spectrometer.

Yield test. First, a series of solution was prepared from 0.05 to $1.5 \mathrm{~mol} \mathrm{~L}^{-1}$. Then, all of the solutions were placed in heated platen until it crystalline completely. The yield curve was obtained by weighing SCs. The yield was calculated by the ratio of the mass of a $\mathrm{MAPbBr}_{3} \mathrm{SC}$ with the solute of $\mathrm{MABr}$ and $\mathrm{PbBr}_{2}$.

Steady-state and time-resolved PL measurements. The TRPL measurements of $\mathrm{MAPbBr}_{3}$ SCs were performed using a HORIBA Jobin Yvon IBH Ltd spectrometer. The steady-state PL spectra were recorded with a fiber optic spectrometer (ideaoptics, NOVA-EX) excited with a $445 \mathrm{~nm}$ CW laser.

SCLC measurements. Current as a function of the applied voltage was measured using a Keysight B2912A Precision Sources, using a rather simple geometry with two electrodes on opposite sides of the sample. Ohmic contacts were deposited on opposite sides of the sample by consecutively thermal evaporation of $\mathrm{C} 60 / \mathrm{Cu}$ for electron-only and Au for hole-only devices. The sample was kept in a dark environment at room temperature. The device area is $0.04 \mathrm{~cm}^{2}$. The thicknesses of $\mathrm{HT}-\mathrm{MAPbBr}_{3}$ single devices are $2.88 \mathrm{~mm}$ for electron-only and $3.02 \mathrm{~mm}$ for holeonly, while those of LDSC-MAPbBr 3 one are $3.12 \mathrm{~mm}$ for electron-only and $3.15 \mathrm{~mm}$ for hole-only, respectively. The thicknesses of the $\mathrm{MAPbBr}_{3} \mathrm{SCs}$ were measured by a digital vernier caliper. A nonlinear response was observed and analyzed according to the SCLC theory.

Device fabrication and measurement. The fabricated device with structure of $\mathrm{InGa} / \mathrm{C} 70 / \mathrm{MAPbBr}_{3} \mathrm{SC} / \mathrm{Au}$. A $35 \mathrm{~nm}$ Au electrons and $50 \mathrm{~nm}$ C70 layer were thermally evaporated under $2.6 \times 10^{-6}$ Torr. The top InGa electrode was coated on the C70 layer. The current-voltage and current-time characteristics of the PDs were measured by a Semiconductor analyzer B1500A under a white LED excitation and $\mathrm{X}$-ray excitation. The X-ray source is a commercially available SPELLMAN XRB011 tube, with a tungsten anode and $20 \mathrm{~W}$ maximum power output. The radiation dose rate was carefully calibrated by using a standard dosimeter (PDM127B-SH). Fast response was obtained using a white LED modulated with an arbitrary wave function generator (Agilent, 33612A), and the photocurrent responses of these devices were recorded with a digital storage oscilloscope (LeCroy Waverunner 8254). The fast response was obtained using an X-ray source with a mechanical shutter, and photocurrent response of those devices were recorded with a semiconductor analyzer B1500A. The active area is $0.095 \mathrm{~cm}^{2}$ of LDSC-MAPbBr SC device and $0.159 \mathrm{~cm}^{2}$ of HT-MAPbBr 3 SC device. The thickness is $2.02 \mathrm{~mm}$ for LDSC-MAPbBr ${ }_{3}$ SC and $1.42 \mathrm{~mm}$ for $\mathrm{HT}-\mathrm{MAPbBr}_{3} \mathrm{SC}$.

\section{Data availability}

The data that support the plots within this paper are available from the corresponding author upon request.

Received: 21 October 2019; Accepted: 16 February 2020;

Published online: 04 March 2020

\section{References}

1. Kim, M. et al. Methylammonium chloride induces intermediate phase stabilization for efficient perovskite solar cells. Joule 3, 1-14 (2019).

2. Jiang, Q. et al. Surface passivation of perovskite film for efficient solar cells. Nat. Photonics 13, 460-466 (2019).

3. Yang, G. et al. Effective carrier-concentration tuning of $\mathrm{SnO}_{2}$ quantum dot electron-selective layers for high-performance planar perovskite solar cells. Adv. Mater. 30, el706023 (2018).

4. Wang, Z. et al. High irradiance performance of metal halide perovskites for concentrator photovoltaics. Nat. Energy 3, 855-861 (2018).
5. Liu, Z. et al. Gas-solid reaction based over one-micrometer thick stable perovskite films for efficient solar cells and modules. Nat. Commun. 9, 3880 (2018).

6. Cao, Y. et al. Perovskite light-emitting diodes based on spontaneously formed submicrometre-scale structures. Nature 562, 249-253 (2018).

7. Lin, K. et al. Perovskite light-emitting diodes with external quantum efficiency exceeding 20 per cent. Nature 562, 245-248 (2018)

8. Xiao, M. et al. Surface ligands stabilized lead halide perovskite quantum dot photocatalyst for visible light-driven hydrogen generation. Adv. Funct. Mater. 29, 1905683 (2019).

9. Jia, Y., Kerner, R. A., Grede, A. J., Rand, B. P. \& Giebink, N. C. Continuouswave lasing in an organic-inorganic lead halide perovskite semiconductor. Nat. Photonics 11, 784-788 (2017).

10. Gui, P. et al. High-performance photodetectors based on single all-inorganic $\mathrm{Cs} \mathrm{PbBr}_{3}$ perovskite microwire. ACS Photonics 5, 2113-2119 (2018).

11. Bao, C. et al. Low-noise and large-linear-dynamic-range photodetectors based on hybrid-perovskite thin-single-crystals. Adv. Mater. 29, 1703209 (2017).

12. Liu, Y. et al. Thinness- and shape-controlled growth for ultrathin singlecrystalline perovskite wafers for mass production of superior photoelectronic devices. Adv. Mater. 28, 9204-9209 (2016).

13. Lin, Q., Armin, A., Burn, P. L. \& Meredith, P. Filterless narrowband visible photodetectors. Nat. Photonics 9, 687-694 (2015).

14. Yao, F. et al. High rubidium-formamidinium ratio perovskites for highperformance photodetection with enhanced stability. ACS Appl. Mater. Interfaces 11, 39875-39881 (2019).

15. Yu, Z. \& Sun, L. Recent progress on hole-transporting materials for emerging organometal halide perovskite solar cells. Adv. Energy Mater. 5, 1500213 (2015).

16. Li, F. et al. A cation-exchange approach for the fabrication of efficient methylammonium tin Iodide perovskite solar cells. Angew. Chem. Int. Ed. $\mathbf{5 8}$ 6688-6692 (2019)

17. Yao, F., Gui, P., Zhang, Q. \& Lin, Q. Molecular engineering of perovskite photodetectors: recent advances in materials and devices. Mol. Syst. Des. Eng. 3, 702-716 (2018).

18. Chao, L. et al. Room-temperature molten salt for facile fabrication of efficien and stable perovskite solar cells in ambient air. Chem 5, 995-1006 (2019).

19. Shi, D. et al. Low trap-state density and long carrier diffusion in organolead trihalide perovskite single crystals. Science 347, 519-522 (2015).

20. Dong, Q. et al. Electron-hole diffusion lengths $>175 \mu \mathrm{m}$ in solution-grown CH3NH3PbI3 single crystals. Science 347, 967-970 (2015).

21. Ball, J. M. \& Petrozza, A. Defects in perovskite-halides and their effects in solar cells. Nat. Energy 1, 16149 (2016).

22. Kawamura, Y., Mashiyama, H. \& Hasebe, K. Structural study on cubic-tetragonal transition of $\mathrm{CH}_{3} \mathrm{NH}_{3} \mathrm{PbI}_{3}$. J. Phys. Soc. Jpn. 71, 1694-1697 (2002).

23. Liu, Y. et al. Fast growth of thin $\mathrm{MAPbI}_{3}$ crystal wafers on aqueous solution surface for efficient lateral-structure perovskite solar cells. Adv. Funct. Mater. 29, 1807707 (2019).

24. Chen, Y. X. et al. General space-confined on-substrate fabrication of thickness-adjustable hybrid perovskite single-crystalline thin films. J. Am. Chem. Soc. 138, 16196-16199 (2016).

25. Liu, Y. et al. Low-temperature-gradient crystallization for multi-inch highquality perovskite single crystals for record performance photodetectors. Mater. Today 22, 67-75 (2019).

26. Yamada, Y. et al. Dynamic optical properties of $\mathrm{CH}_{3} \mathrm{NH}_{3} \mathrm{PbI}_{3}$ single crystals as revealed by one- and two-photon excited photoluminescence measurements. J. Am. Chem. Soc. 137, 10456-10459 (2015)

27. Saidaminov, M. I., Abdelhady, A. L., Maculan, G. \& Bakr, O. M. Retrograde solubility of formamidinium and methylammonium lead halide perovskites enabling rapid single crystal growth. Chem. Commun. 51, 17635-17778 (2015).

28. Liu, Y. et al. Two-inch-sized perovskite $\mathrm{CH}_{3} \mathrm{NH}_{3} \mathrm{PbX}_{3}(\mathrm{X}=\mathrm{Cl}, \mathrm{Br}$, I) crystals: growth and characterization. Adv. Mater. 27, 5176-5183 (2015).

29. Dang, Y. et al. Bulk crystal growth of hybrid perovskite material $\mathrm{CH}_{3} \mathrm{NH}_{3} \mathrm{PbI}_{3}$. Crystengcomm 17, 665-670 (2015).

30. Stoumpos, C. C. et al. Crystal growth of the perovskite semiconductor $\mathrm{CsPbBr}$ : a new material for high-energy radiation detection. Cryst. Growth Des. 13, 2722-2727 (2013).

31. Peng, W. et al. Solution-grown monocrystalline hybrid perovskite films for hole-transporter-free solar cells. Adv. Mater. 28, 3383-3390 (2016).

32. Chen, Z. et al. Single-crystal $\mathrm{MAPbI}_{3}$ perovskite solar cells exceeding $21 \%$ power conversion efficiency. ACS Energy Lett. 4, 1258-1259 (2019).

33. Liu, Y. et al. 20-mm-large single-crystalline formamidinium-perovskite wafer for mass production of integrated photodetectors. Adv. Opt. Mater. 4, 1829-1837 (2016)

34. Liu, Y. et al. $120 \mathrm{~mm}$ single-crystalline perovskite and wafers: towards viable applications. Sci. China-Chem. 60, 1367-1376 (2017). 
35. Wei, H. et al. Dopant compensation in alloyed $\mathrm{CH}_{3} \mathrm{NH}_{3} \mathrm{PbBr}_{3-\mathrm{x}} \mathrm{Cl}_{\mathrm{x}}$ perovskite single crystals for gamma-ray spectroscopy. Nat. Mater. 16, 826-833 (2017).

36. Wei, W. et al. Monolithic integration of hybrid perovskite single crystals with heterogenous substrate for highly sensitive X-ray imaging. Nat. Photonics 11, 315-321 (2017).

37. Nayak, P. K. et al. Mechanism for rapid growth of organic-inorganic halide perovskite crystals. Nat. Commun. 7, 13303 (2016).

38. Saidaminov, M. I. et al. High-quality bulk hybrid perovskite single crystals within minutes by inverse temperature crystallization. Nat. Commun. 6, 7586 (2015).

39. Ono L. K., Liu S., Qi Y. Reducing detrimental defects for high-performance metal halide perovskite solar cells. Angew. Chem. Int. Ed. https://doi.org/ 10.1002/anie.201905521 (2020)

40. Ohmann, R. et al. Real-space imaging of the atomic structure of organic-inorganic perovskite. J. Am. Chem. Soc. 137, 16049-16054 (2015).

41. $\mathrm{Ku}, \mathrm{Z}$. et al. Solvent engineering for fast growth of centimetric high-quality $\mathrm{CH}_{3} \mathrm{NH}_{3} \mathrm{PbI}_{3}$ perovskite single crystals. N. J. Chem. 40, 7261-7264 (2016).

42. Chen, J. et al. A ternary solvent method for large-sized two-dimensional perovskites. Angew. Chem. Int. Ed. 56, 2390-2394 (2017).

43. Maculan, G. et al. $\mathrm{CH}_{3} \mathrm{NH}_{3} \mathrm{PbCl}_{3}$ single crystals: inverse temperature crystallization and visible-blind UV-photodetector. J. Phys. Chem. Lett. 6, 3781-3786 (2015).

44. Zhou, G., Li, M., Zhao, J., Molokeev, M. S. \& Xia, Z. Single-component whitelight emission in 2D hybrid perovskites with hybridized halogen atoms. Adv. Opt. Mater. 7, 1901335 (2019).

45. Sung, M.-H., Kim, J.-S., Kim, W.-S., Hirasawa, I. \& W.-S., K. Modification of crystal growth mechanism of yttrium oxalate in metastable solution. J. Cryst. Growth 235, 529-540 (2002).

46. LaMer, V. K. DRH. Theory, production and mechanism of formation of monodispersed hydrosols. J. Am. Chem. Soc. 72, 4847-4854 (1950).

47. LaMer, V. K. Nucleation in phase transitions. Ind. Eng. Chem. 44, 1270-1277 (1952).

48. Jung, M., Ji, S. G. \& KimG., I. S. S. Perovskite precursor solution chemistry: from fundamentals to photovoltaic applications. Chem. Soc. Rev. 48, 2011-2038 (2019).

49. KP, H. Importance of the two-step crystal growth model. Chem. Eng. Sci. 40, 641-646 (1985).

50. Nancollas, G. H. Kinetics of crystal growth from solution. J. Cryst. Growth 3, 335-339 (1968).

51. Liu, Y. et al. A $1300 \mathrm{~mm}^{2}$ ultrahigh-performance digital imaging assembly using high-quality perovskite single crystals. Adv. Mater. 30, 1707314 (2018).

52. Poglitsch, A. \& Weber, D. Dynamic disorder in methylammoniumtrihalogenoplumbates (II) observed by millimeter-wave spectroscopy. J. Chem. Phys. 87, 6373-6378 (1987).

53. Ayres, J. R. Characterization of trapping states in polycrystalline-silicon thin film transistors by deep level transient spectroscopy. J. Appl. Phys. 74, 1787-1792 (1993).

54. Kerr, L. L. et al. Investigation of defect properties in $\mathrm{Cu}(\mathrm{In}, \mathrm{Ga}) \mathrm{Se}_{2}$ solar cells by deep-level transient spectroscopy. Solid-State Electron. 48, 1579-1586 (2004).

55. Balcioglu, A., Ahrenkiel, R. K. \& Hasoon, F. Deep-level impurities in CdTe/ CdS thin-film solar cells. J. Appl. Phys. 88, 7175-7178 (2000).

56. Ahmadi, M., Wu, T. \& Hu, B. A review on organic-inorganic halide perovskite photodetectors: device engineering and fundamental physics. $A d v$. Mater. 29, 1605242 (2017).

57. Yong Churl, Kim et al. Printable organometallic perovskite enables large-area, low-dose X-ray imaging. Nature 550, 87-91 (2017).
58. Choquett, Martin et al. Direct selenium x-ray detector for fluoroscopy, R\&F, and radiography. Proc. SPIE 3977, 128-136 (2000).

\section{Acknowledgements}

G.F. acknowledge the support of the National Natural Science Foundation of China (Grant No. 11674252) and the Natural Science Foundation of Hubei Province, China (Grant No. 2019AAA020), Q.L. acknowledge the support of the National Natural Science Foundation of China (Grant No. 61875154), the Natural Science Foundation of Hubei Province, China (Grant No. 2018CFA021) and the Fundamental Research Funds for the Central Universities (Grant No. 2042018kf0207). C.T. acknowledge the support of the National Natural Science Foundation of China (Grant No. 61904126), the Natural Science Foundation of Hubei Province, China (Grant No. 2019CFB122) and the Fundamental Research Funds for the Central Universities (Grant No. 2042019kf0042).

\section{Author contributions}

G.F., Q.L., C.T. supervised the project during the whole process of the experiment. J.P. and Q.L. measured the $I-V$ and detectors properties. R.L. and Q.L. measured and analyzed the PL. W.L and C.L. measured and analyzed the XRD. P.G. and B.L. analyzed the pole figure. F.Y. proposed the idea, designed the experiment, performed all other measurements, and wrote the paper. C.T., Q.L., and G.F. revised the paper.

\section{Competing interests}

The authors declare no competing interests.

\section{Additional information}

Supplementary information is available for this paper at https://doi.org/10.1038/s41467020-15037-x.

Correspondence and requests for materials should be addressed to C.T., Q.L. or G.F.

Peer review information Nature Communications thanks Paul Evans and other, anonymous, reviewers for their contributions to the peer review of this work.

Reprints and permission information is available at http://www.nature.com/reprints

Publisher's note Springer Nature remains neutral with regard to jurisdictional claims in published maps and institutional affiliations.

\begin{abstract}
cC) (i) Open Access This article is licensed under a Creative Commons By Attribution 4.0 International License, which permits use, sharing, adaptation, distribution and reproduction in any medium or format, as long as you give appropriate credit to the original author(s) and the source, provide a link to the Creative Commons license, and indicate if changes were made. The images or other third party material in this article are included in the article's Creative Commons license, unless indicated otherwise in a credit line to the material. If material is not included in the article's Creative Commons license and your intended use is not permitted by statutory regulation or exceeds the permitted use, you will need to obtain permission directly from the copyright holder. To view a copy of this license, visit http://creativecommons.org/ licenses/by/4.0/.
\end{abstract}

(c) The Author(s) 2020 